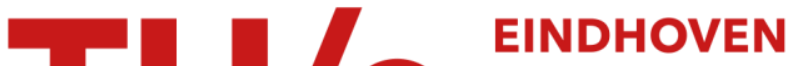 UNIVERSITY OF TECHNOLOGY
}

\section{Modeling of the transient mobility in disordered organic semiconductors with a Gaussian density of states}

\section{Citation for published version (APA):}

Germs, W. C., Holst, van der, J. J. M., Mensfoort, van, S. L. M., Bobbert, P. A., \& Coehoorn, R. (2011). Modeling of the transient mobility in disordered organic semiconductors with a Gaussian density of states. Physical Review B, 84(16), 165210-1/7. [165210]. https://doi.org/10.1103/PhysRevB.84.165210

DOI:

10.1103/PhysRevB.84.165210

Document status and date:

Published: 01/01/2011

\section{Document Version:}

Publisher's PDF, also known as Version of Record (includes final page, issue and volume numbers)

\section{Please check the document version of this publication:}

- A submitted manuscript is the version of the article upon submission and before peer-review. There can be important differences between the submitted version and the official published version of record. People interested in the research are advised to contact the author for the final version of the publication, or visit the $\mathrm{DOI}$ to the publisher's website.

- The final author version and the galley proof are versions of the publication after peer review.

- The final published version features the final layout of the paper including the volume, issue and page numbers.

Link to publication

\section{General rights}

Copyright and moral rights for the publications made accessible in the public portal are retained by the authors and/or other copyright owners and it is a condition of accessing publications that users recognise and abide by the legal requirements associated with these rights.

- Users may download and print one copy of any publication from the public portal for the purpose of private study or research.

- You may not further distribute the material or use it for any profit-making activity or commercial gain

- You may freely distribute the URL identifying the publication in the public portal.

If the publication is distributed under the terms of Article 25fa of the Dutch Copyright Act, indicated by the "Taverne" license above, please follow below link for the End User Agreement:

www.tue.nl/taverne

Take down policy

If you believe that this document breaches copyright please contact us at:

openaccess@tue.nl

providing details and we will investigate your claim. 


\title{
Modeling of the transient mobility in disordered organic semiconductors with a Gaussian density of states
}

\author{
W. Chr. Germs, ${ }^{1,2, *}$ J. J. M. van der Holst, ${ }^{3}$ S. L. M. van Mensfoort,,${ }^{1,2}$ P. A. Bobbert, ${ }^{3}$ and R. Coehoorn ${ }^{1,2}$ \\ ${ }^{1}$ Molecular Materials and Nanosystems, Department of Applied Physics, Eindhoven University of Technology, \\ P.O. Box 513, NL-5600 MB Eindhoven, The Netherlands \\ ${ }^{2}$ Philips Research Laboratories, High Tech Campus 4, NL-5656 AE Eindhoven, The Netherlands \\ ${ }^{3}$ Group Polymer Physics, Eindhoven Polymer Laboratories and Dutch Polymer Institute, Eindhoven University of Technology, P.O. Box 513, \\ NL-5600 MB Eindhoven, The Netherlands
}

(Received 20 September 2011; published 25 October 2011; publisher error corrected 31 October 2011)

\begin{abstract}
The charge-carrier mobility in organic semiconductors is often studied using non-steady-state experiments. However, energetic disorder can severely hamper the analysis due to the occurrence of a strong time dependence of the mobility caused by carrier relaxation. The multiple-trapping model is known to provide an accurate description of this effect. However, the value of the conduction level energy and the hopping attempt rate, which enter the model as free parameters, are not a priori known for a given material. We show how for the case of a Gaussian density of states both parameters can be deduced from the parameter values used to describe the measured dc current-voltage characteristics within the framework of the extended Gaussian disorder model. The approach is validated using three-dimensional Monte Carlo modeling. In the analysis, the charge-density dependence of the time-dependent mobility is included. The model is shown to successfully predict the low-frequency differential capacitance of sandwich-type devices based on a polyfluorene copolymer.
\end{abstract}

DOI: $10.1103 /$ PhysRevB.84.165210

PACS number(s): 72.80.Le, 73.61.Ph, 85.30.De

\section{INTRODUCTION}

The organic semiconductors which are used in organic light-emitting diodes (OLEDs) and organic field-effect transistors are often strongly disordered. The charge transport is then due to hopping between states which are localized on a molecule or on a conjugated segment in a polymer. Energetic disorder can give rise to a strong temperature $(T)$, electric field $(F)$, and charge-carrier density $(n)$ dependence of the charge-carrier mobility $(\mu)$, depending on the detailed shape of the density of states (DOS). ${ }^{1-5}$ However, the mobility function $\mu(T, F, n)$ is only well defined under steady-state conditions. The transient electrical response can be severely affected by charge-carrier relaxation effects, caused by the slow and widely time-distributed hopping processes in between high-energy "conduction" states and low-energy states in the tail of the DOS which effectively act as trap states. ${ }^{6}$ Wellknown consequences are the anomalous transit-time dispersion observed in time-of-flight (TOF) mobility measurements ${ }^{7-11}$ or in transient photocurrent measurements, ${ }^{12,13}$ an anomalous low-frequency electrical admittance, ${ }^{14-16}$ and hysteresis or even long-time memory effects. ${ }^{17}$

Transient effects in energetically disordered semiconductors have been studied by Monte Carlo modeling ${ }^{1,18}$ and using the multiple-trapping (MT) model, ${ }^{6,14,19-27}$ which is better suitable for long time scales or at low frequencies. Within the MT model, the transport is assumed to be due to the fraction of carriers in a high-energy conduction level $\left(E_{\mathrm{c}}\right)$, while the remaining carriers reside in a distribution of low-lying localized states in which they are immobile. The effect of a sudden spatially uniform increase of the carrier density is described as an instantaneous increase of the population of the conduction level, followed by multiple capture-and-release processes during which the average carrier energy decreases. The time dependence of the mobility is assumed to be equal to the average time fraction during which carriers reside at the conduction level times the mobility of carriers in the conduction level. The energetic relaxation process leads to a decrease of the mobility, in a manner which depends on the detailed shape of the DOS. For the case of an exponential DOS, no characteristic time constant is involved in the relaxation process, as it evolves to energetically ever deeper states. The DOS is then proportional to $\exp \left[E / \varepsilon_{0}\right]$, with $E$ the energy and $\varepsilon_{0} \equiv k_{\mathrm{B}} T_{0}$ a characteristic energy, where $k_{\mathrm{B}}$ is the Boltzmann constant and $T_{0}$ is a characteristic temperature. If $T \leqslant T_{0}$, the time- $(t)$ and temperature-dependent $(T)$ mobility is to a good approximation proportional to $t^{\alpha-1}$, with $\alpha=T / T_{0} \cdot{ }^{20,21} \mathrm{In}$ contrast, for the case of a Gaussian DOS with a width (standard deviation) $\sigma$, the time dependence of the mobility is more complex and the relaxation process ends when the average carrier energy is equal to the thermal equilibrium energy, $E_{0}=-\sigma^{2} /\left(k_{\mathrm{B}} T\right){ }^{1}$ The mobility relaxation time depends sensitively on the position of the conduction level with respect to the thermal equilibrium energy, ${ }^{24,25}$ viz. as

$$
t_{0, \mathrm{mob}}=\frac{1}{v_{0}} \exp \left(\frac{E_{\mathrm{c}}-E_{0}}{k_{\mathrm{B}} T}\right),
$$

with $v_{0}$ the hopping attempt frequency. The concept of a conduction (or "transport") energy is well established in theories of the steady-state mobility ${ }^{24,28-30}$ and provides an excellent description of its carrier density dependence. ${ }^{30,31}$ However, it has been defined in various ways and it is not known which expression for $E_{\mathrm{c}}$ should be used when modeling relaxation processes. Furthermore, the hopping attempt frequency, which determines the capture-and-release rates, is in general not known. Therefore, MT models can so far only empirically describe charge-carrier relaxation in materials with a Gaussian DOS.

A second consequence of the multiple-trapping process, which becomes apparent when the charge carriers are introduced only in a spatially strongly confined region and in the 
presence of an electric field, is an anomalous broadening of the initially narrow package of charge carriers, leading to a diffusion coefficient along the field direction that is much larger than that expected from an application of the Einstein relation. This so-called anomalous dispersive transport effect may be understood as a result of the fact that at any time $t$ those carriers which have been captured in deep trap states, defined as states from which the release time is (much) larger than $t$, are essentially immobile so that equilibrium statistics is not applicable. A thermal equilibrium distribution has only been established (after multiple-trapping and release processes) for those carriers which reside in shallow trap states, defined as states from which the release time is (much) smaller than $t$. This "demarcation-line picture,"20,21 involving a time-dependent energy which separates the shallow and deep trap states, has been found to provide a convenient basis for calculating the effects of the time-dependent mobility and anomalous dispersion on the transient current measured in TOF measurements. Measurements of the post-transit-time photocurrent response in such experiments may even be used to determine the shape of the deep part of the DOS, i.e., the part of the DOS within which no thermal equilibrium occupation has been established yet at the transit time. ${ }^{23}$ Again, also dispersive transport manifests itself in qualitatively different ways for the cases of an exponential DOS and a Gaussian DOS, viz. as a continuing process and a process which ends after a time

$$
t_{0, \text { disp }}=\frac{1}{v_{0}} \exp \left(\frac{E_{\mathrm{c}}-2 E_{0}}{k_{\mathrm{B}} T}\right),
$$

respectively, as shown by Nikitenko et al..$^{25}$ The dispersion process will thus in general proceed long after the mobility has been relaxed. As already suggested by Eq. (2), Nenashev et $a l .{ }^{26}$ showed recently theoretically that the most important sites determining this effect reside around the energy $2 E_{0}=$ $-2 \sigma^{2} /\left(k_{\mathrm{B}} T\right)$ and that the effect almost disappears if sites with energies below the thermal equilibrium energy $E_{0}$ are eliminated. On the other hand, the mobility was found to be only weakly affected by taking away states below $E_{0}$. As the anomalous dispersion effect is thus sensitive to the presence of a very small fraction of deep tail states, it is expected to be less pronounced in small systems, ${ }^{26}$ e.g., systems containing thin layers, such as OLEDs. However, this has not been studied systematically. Relaxation effects have so far most intensively been studied for relatively thick devices, such as used in TOF experiments (typically $10 \mu \mathrm{m}$ ).

As far as is known to the authors, all TOF analyses of relaxation and anomalous dispersive transport and all theoretical studies were carried out under the assumption that the carriers may be viewed as noninteracting. For the case of a Gaussian DOS, this corresponds to considering only systems with carrier concentrations within the Boltzmann regime. Whereas this is often a fair approximation when analyzing TOF experiments, a significant carrier density dependence of the transient charge transport processes is expected under space-charge-limited current (SCLC) transport conditions.

In this paper, we further develop the MT model for the case of semiconductors with a Gaussian DOS and show how it can be used to predictively model relaxation, viz. by (i) applying the MT formalism to relaxation at any arbitrary carrier density, also outside the Boltzmann regime, (ii) providing for $E_{\mathrm{c}}$ a simple relationship with $\sigma$, and (iii) providing for $\nu_{0}$ a simple relationship with $\sigma$ and the hopping site density $N_{\mathrm{t}}$. Both parameters $\sigma$ and $N_{\mathrm{t}}$ can already be obtained from steady-state current density $(J)$ versus voltage $(V)$ measurements. Therefore, no additional parameters are involved. The expression for $E_{\mathrm{c}}$ is obtained from three-dimensional (3D) Monte Carlo calculations and is shown to be in good agreement with the results obtained recently independently from percolation theory. ${ }^{32}$ The approach is applicable to organic and inorganic semiconductors. We discuss how it leads to a time- or frequency-dependent mobility that can be used to model transients measured in any experiment, and we apply it to the case of the differential capacitance $(C)$ of hole-only metal-organic semiconductormetal (MOM) devices based on a blue-emitting copolymer. The devices studied operate under SCLC conditions, and the development and application of a charge-carrier densitydependent model for the ac mobility is shown to be relevant.

In Sec. II, the structure of the devices studied is presented, and it is discussed why measuring the angular frequency $(\omega)$ dependence of $C(V)$ characteristics of MOM devices is expected to be a particularly sensitive method for probing relaxation effects, as suggested earlier in Refs. 14-16. Section III contains the main results, including an expression for the hopping attempt frequency, the conduction energy level for materials with a Gaussian DOS, and expressions for the ac mobility. In Sec. IV an analysis is presented of the measured layer-thickness-dependent $C(V)$ curves, which provides support for the method developed. Section $\mathrm{V}$ gives a summary and conclusions.

\section{DEVICE STRUCTURE}

The devices studied are based on a blue-emitting polyfluorene-(7.5 mol \% triarylamine) (PF-TAA) co-polymer, supplied by Sumation Co., of which the molecular structure is shown in Fig. 1(a). The layer structure of the devices is

$$
\text { glass | ITO | PEDOT:PSS | PF-TAA | Pd, }
$$

with an indium tin oxide (ITO)/100-nm poly(3,4-ethylenedioxythiophene)poly(styrene sulphonic acid) (PEDOT:PSS) anode and a 100-nm palladium cathode. Details on the device fabrication and measurement methods are given in Ref. 34 .

In PF-TAA, the hole transport takes place by hopping in between states localized on the TAA units. From an analysis of the steady-state $J(V)$ curves, measured for a range of temperatures and PF-TAA layer thicknesses, it was found that the hole mobility of PF-TAA can be accurately described by assuming hopping transport in between spatially uncorrelated localized states with a Gaussian DOS. ${ }^{35,36}$ The carrier density and electric field dependence of the steady-state mobility are then given by the extended Gaussian disorder model (EGDM), ${ }^{4}$ and described by three parameters, viz. the width $\sigma \cong 0.13 \mathrm{eV}$ of the Gaussian DOS, the hopping site density $N_{\mathrm{t}} \cong 0.6 \times 10^{27} \mathrm{~m}^{-3}$, and the mobility in the limit of zero carrier density and zero field, $\mu_{0}(295 \mathrm{~K})=$ $5.2 \times 10^{-12} \mathrm{~m}^{2} /(\mathrm{V} \mathrm{s})$. The temperature dependence of $\mu_{0}(T)$ is found to be described well by

$$
\mu_{0}(T)=\mu_{0, \mathrm{EGDM}}^{*} \exp \left(-\chi \hat{\sigma}^{2}\right)
$$


(a)

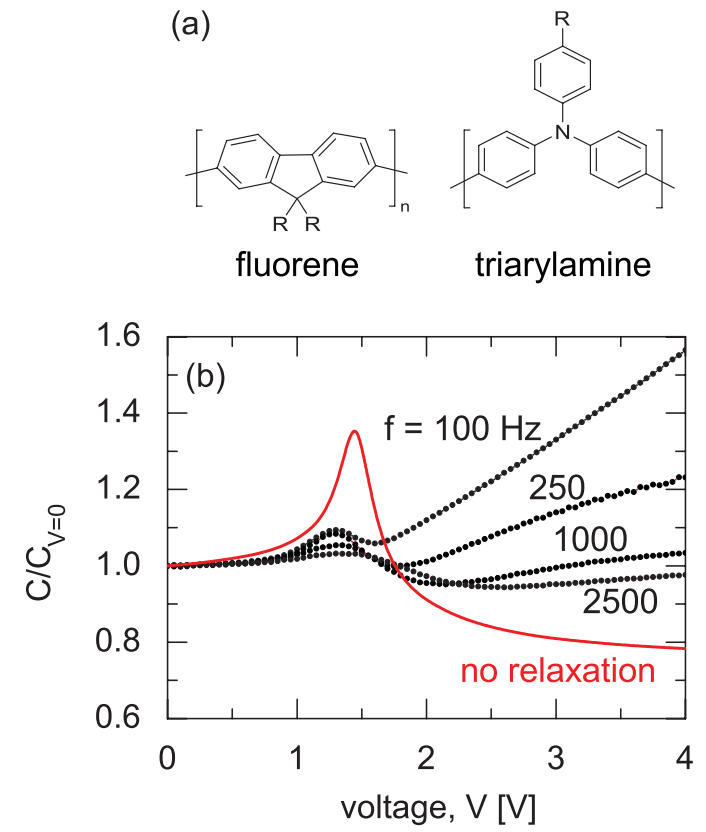

FIG. 1. (Color online) (a) Schematic structure of PF-TAA. (b) Experimental $C(V) / C_{V=0}$ curves (symbols) for a $97-\mathrm{nm}$ device at $T=295 \mathrm{~K}$ for various frequencies ${ }^{33}$ and calculated $100-\mathrm{Hz}$ curve without relaxation.

with $\hat{\sigma} \equiv \sigma /\left(k_{\mathrm{B}} / T\right)$ and where $\chi$ is in the range as expected within the scope of the EGDM, viz. $\chi \cong 0.39$. In the high-temperature and low-carrier-density limit, the zero-field mobility is equal to $\mu_{0, \mathrm{EGDM}}^{*}=1.4 \times 10^{-7} \mathrm{~m}^{2} /(\mathrm{V} \mathrm{s})$. The hole injection barriers at the anode and cathode are 0 and $1.9 \mathrm{eV}$, respectively. ${ }^{35,37}$

The capacitance-voltage curves shown in Fig. 1(b) may be understood as follows. In Ref. 34, it was discussed how the voltage and height of a distinct low-frequency peak in the experimental $C(V)$ curves can be used to determine the injection barriers at both interfaces. The peak voltage, $1.30 \pm 0.05 \mathrm{~V}$, may be viewed as an effective value of the conductance onset voltage. ${ }^{38}$ It is significantly smaller than the built-in voltage, $V_{\mathrm{bi}} \cong 1.9 \mathrm{~V}$, as a result of the space charge near the anode due to charge-carrier diffusion. The full $C(V)$ curve shows the result of a calculation for a frequency $f=100 \mathrm{~Hz}$, using the $f$-independent EGDM mobility function. ${ }^{39}$ The calculated peak voltage, $1.40 \pm 0.10 \mathrm{~V}$, agrees well with the experimental value. However, the simulation predicts at high voltages a $V$-independent capacitance smaller than $C_{V=0}$, resulting from the delayed response of the space charge in the bulk of the device, whereas the experimental $C(V)$ curves show a pronounced $f$-dependent increase. A similar effect has been observed earlier for various other organic semiconductor systems and has been interpreted either as an intrinsic effect, resulting from the charge-carrier relaxation in the presence of energetic disorder, ${ }^{15,40}$ or due to the presence of extrinsic trap states. ${ }^{16,41,42}$ Martens et al. observed the effect for devices based on poly ( $p$-phenylene vinylene), attributed it to charge-carrier relaxation within the Gaussian DOS, and analyzed the admittance in terms of a complex mobility of the form

$$
\mu(\omega)=\mu_{\mathrm{dc}}\left[1+M\left(i \omega \tau_{\mathrm{t}}\right)^{1-\alpha}\right],
$$

with $\mu_{\mathrm{dc}}$ the dc mobility, $\tau_{\mathrm{t}}$ the layer-thickness- and voltagedependent average charge-carrier transit time, and $\alpha$ and $M$ phenomenological parameters. ${ }^{15}$ This approach was also adopted in more recent work. ${ }^{40}$ However, no relationship was established between these parameters and the shape of the DOS. We show that it is indeed possible to define a frequency-dependent mobility but that it only depends on the EGDM parameters $\sigma, N_{\mathrm{t}}$, and $\mu_{0, \mathrm{EGDM}}^{*}$ and on the local carrier density, and not on the layer thickness and voltage.

\section{MODELING THE AC MOBILITY}

\section{A. Hopping attempt frequency $v_{0}$}

Within the MT model, the relaxation dynamics is modeled as a local process, i.e., neglecting the spatial distribution of the sites, using a total DOS composed of a normalized Gaussian DOS with a width equal to the experimental value and a normalized $\delta$-function-shaped conduction level. Figure 2 shows schematically the effect of relaxation on the current density $J(t)$ [Fig. 2(a)] due to a sudden small increase of the carrier density [Fig. 2(b)]. Initially, $J$ is strongly enhanced, as all additional carriers occupy then the highenergy conduction level [Fig. 2(c)]. Upon relaxation, the occupation probability $p_{\mathrm{c}}$ of the conduction states decreases to the dynamic equilibrium value. The current density is proportional to $p_{\mathrm{c}}(t)$. The final current density is larger than the initial current density $(t<0)$ due to the larger final carrier density and, depending on the initial carrier density $n_{0}$, due to a density dependence of the mobility. The relaxation kinetics is described as follows. For downward hops (capture), the rate is equal to $r_{\text {cap }}=v_{0}\left(1-p_{\mathrm{f}}\right)$, with $v_{0}$ an attempt frequency and $p_{\mathrm{f}}$ the occupation probability of the final state. The rate of upward hops to $E_{\mathrm{c}}$ (release, a thermally activated process) is given by $r_{\text {rel }}=v_{0} \exp \left(-\Delta E / k_{\mathrm{B}} T\right)$, with $\Delta E$ the energy difference between the final and initial states. The energy-difference dependence of the rates is thus equal to that given by the Miller-Abrahams expression ${ }^{43}$ used in 3D master-equation (3D-ME) modeling of the mobility. ${ }^{4}$ Within our model, $v_{0}$ is taken to be equal to the value for nearest-neighbor (NN) hops in the 3D-ME model, ${ }^{44}$

$$
v_{0, \mathrm{NN}} \cong \mu_{0, \mathrm{EGDM}}^{*} \frac{\sigma}{a^{2} e},
$$

(a)

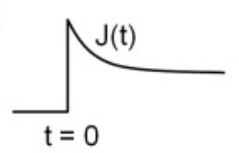

(c)

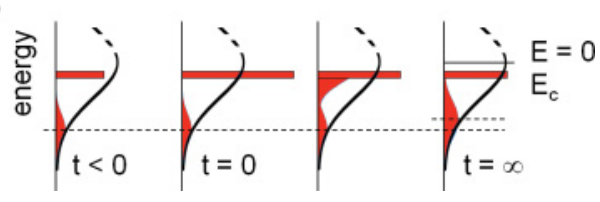

FIG. 2. (Color online) Schematic current density response in (a) a spatially uniform system to (b) a small sudden increase of the carrier density, and (c) a schematic representation of the time-dependent population of the conduction level and the Gaussian DOS. The dashed lines indicate the initial and final positions of the top of the density of occupied states. 


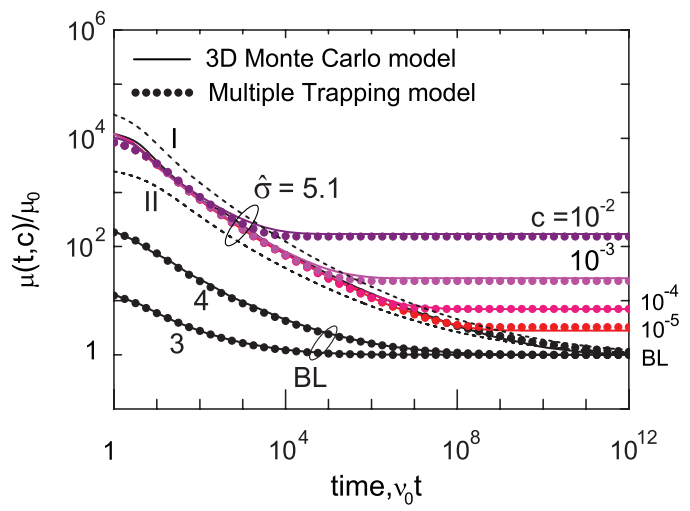

FIG. 3. (Color online) Calculated time dependence of the zerofield mobility relative to $\mu_{0}$ (the dc mobility in the Boltzmann limit, BL) for PF-TAA ( $\hat{\sigma}=5.1$, including results for various concentrations) and for systems with $\hat{\sigma}=4$ and 3 (BL). Results from the MT model (solid spheres) with $E_{\mathrm{c}}=-0.75 \sigma,-0.65 \sigma$, and $-0.60 \sigma$, respectively, are compared with the results from MC calculations (solid curves, smoothened). The dashed curves give the MT results for $\hat{\sigma}=5.1$ (BL) with $E_{\mathrm{c}}=-0.5 \sigma$ (I) and $-1.0 \sigma$ (II). For PF-TAA, the time range corresponds to $0.08 \mathrm{~ns}$ to $80 \mathrm{~s}$.

with $a=N_{\mathrm{t}}^{-1 / 3}$ the average intersite distance and $e$ the elementary charge. The parameter values of $\sigma, N_{\mathrm{t}}$, and $\mu_{0, \mathrm{EGDM}}^{*}$ describing the mobility in PF-TAA, obtained from Ref. 35 and given in Sec. II, yield $v_{0} \cong 1.3 \times 10^{10} \mathrm{~s}^{-1}$ at room temperature.

\section{B. Conduction-level energy $\boldsymbol{E}_{\mathrm{c}}$}

We have determined $E_{\mathrm{c}}$ by first carrying out a 3D-MC calculation of the time dependence of the zero-field mobility in the Boltzmann regime. Single carriers are randomly injected at $t=0$ at sites with $E>0$ in an otherwise empty cubic grid with periodic boundary conditions, containing $250^{3} \cong 1.6 \times$ $10^{7}$ sites with a Gaussian energy distribution. By ensemble averaging, a relative accuracy of better than $10 \%$ was realized. Figure 3 (solid curves) shows the resulting time-dependent mobility for various values of $\hat{\sigma} \equiv \sigma /\left(k_{\mathrm{B}} T\right)$. For PF-TAA, with $\sigma=0.13 \mathrm{eV}$, we have $\hat{\sigma}=5.1$ at room temperature. Shortly after $t=0$ the mobility is enhanced by a factor of $\sim 10^{4}$ as compared to the dc mobility in the Boltzmann limit, independent of the carrier density. In the Boltzmann regime, the entire relaxation process takes more than a second. However, at higher concentrations the steady state is reached more quickly. The approach includes thus in a natural way a decrease of the relaxation time with increasing carrier concentration $\left(c \equiv n / N_{\mathrm{t}}\right)$ outside the independent-particle Boltzmann regime, ${ }^{30}$ i.e., when $c>\frac{1}{2} \exp \left(-\frac{1}{2} \hat{\sigma}^{2}\right) \approx 10^{-6}$ for $\hat{\sigma}=5.1$. The effect of relaxation decreases strongly with decreasing disorder, as may be seen from Fig. 3 for $\hat{\sigma}=3$ and 4 (Boltzmann limit). For all cases an excellent fit to the Monte Carlo (MC) curves could be obtained using the MT model, using

$$
\begin{gathered}
E_{\mathrm{c}}^{\mathrm{MC}}(\hat{\sigma}=3)=-(0.60 \pm 0.1) \sigma, \\
E_{\mathrm{c}}^{\mathrm{MC}}(\hat{\sigma}=4)=-(0.65 \pm 0.1) \sigma, \\
E_{\mathrm{c}}^{\mathrm{MC}}(\hat{\sigma}=5.1)=-(0.70 \pm 0.1) \sigma .
\end{gathered}
$$

For the last case, the figure shows the MT results for $E_{\mathrm{c}}=$ $-0.75 \sigma$, and it includes for comparison also results for $-0.5 \sigma$ and $-1.0 \sigma$ as obtained for the Boltzmann limit. It may be seen that the mobility enhancement is very sensitive to the precise value of the conduction-level energy, making it possible to deduce $E_{\mathrm{c}}$ with an uncertainty of only $\pm 0.1 \sigma$.

It is of interest to compare these values for the effective conduction-level energy with the results of a recent analysis by Cottaar et $_{\text {al }}{ }^{32}$ of the transport in systems with a Gaussian DOS using percolation theory. The authors determined for various types of lattices and expressions for the hopping rates the energy $E_{\text {crit }}$ of the critical sites, i.e., the sites with the highest energies which still contribute to the percolating path. For the case of simple cubic systems within which the hopping is described by Miller-Abrahams hopping rates (as in the Monte Carlo carried out in our work), it was found that $E_{\text {crit }}=-0.49 \sigma$, very close to the values of $E_{\mathrm{c}}$ obtained independently in our work from an analysis of the relaxation process. The finding of an effective conductionlevel energy and a critical energy which are both essentially independent of temperature is consistent with our observation that in the discrete lattice systems studied nearest-neighbor hopping processes are predominant, even for PF-TAA with $\hat{\sigma}=5.1$.

The work of Cottaar et al. shows that the critical energy depends on the type of lattice considered. For a face centered cubic (fcc) lattice it is lower than for a simple cubic (sc) lattice, viz., $-0.84 \sigma{ }^{32}$ Such a decrease may be understood qualitatively from percolation theory, from which it follows that in order to establish a percolating pathway each site must have on average at least a certain critical number of nearest neighbors to which it can be connected, i.e. with an energy below $E_{\text {crit }}$. In an fcc lattice, within which each site has 12 nearest neighbors, this criterion is already fulfilled for a lower value of $E_{\text {crit }}$ than for a sc lattice, within which each site has six nearest neighbors. As a consequence, one may expect that also the conduction-level energy for a material depends slightly on the (average) coordination number of the molecules. A study of this effect and more generally of the precise relationship between $E_{\mathrm{c}}$ and $E_{\text {crit }}$ is beyond the scope of the present paper. However, we note that it may be possible to obtain relevant experimental information about this issue from the temperature dependence of the mobility in the zero-field and zero-density limit, given by Eq. (3). Cottaar et al. found that in the range $2 \leqslant \hat{\sigma} \leqslant 6$ the results for Miller-Abrahams hopping on sc and fcc lattices can be well described with $\chi=0.44$ and $\chi=0.40$, respectively. The smaller $\chi$ value for the fcc case arises from the somewhat smaller effective activation energy, resulting from the somewhat lower (more negative) value of the critical energy. For PF-TAA, with $\chi=0.39 \pm 0.01$ as discussed in Sec. II, it would be suggested by the formalism discussed above that the effective coordination number is relatively large and close to that in an fcc lattice.

We finally note that our results concerning $E_{\mathrm{c}}$ are also applicable to systems in which a small low-energy trap (guest) DOS is superimposed on the Gaussian (host) DOS, as observed frequently in electron-transporting materials or in the emissive layers in OLEDs, since it follows from percolation theory that their presence does not affect $E_{\mathrm{c}} .{ }^{30}$ 


\section{Time- and frequency-dependent mobility $\mu(t)$ and $\mu_{\text {ac }}(\omega)$}

The expressions given for $r_{\text {cap }}, r_{\text {rel }}$, and $E_{\mathrm{c}}$ make it possible to calculate $J(t)$ for any transient experiment, under the assumption that the time dependence is predominantly due to the time dependence of the mobility resulting from charge-carrier relaxation. We neglect here the effect of dispersive transport, related to the formation of a nonequilibrium occupation of deep trap states (see Sec. I), which is relevant for large layer thicknesses but which is likely to be of less relevance to the relatively thin devices investigated in this study. The time-dependent mobility is equal to

$$
\mu(t)=\mu_{0, \mathrm{EGDM}} f(F) \frac{\eta_{\mathrm{c}}(t)}{\eta_{\mathrm{c}, \mathrm{BL}}},
$$

with $\mu_{0, \text { EGDM }}$ the dc mobility for $F=0$ and $n=0, f(F)$ the field-dependent mobility enhancement in a Gaussian DOS (from Ref. 4), and $\eta_{\mathrm{c}}(t) \equiv p_{\mathrm{c}}(t) N_{\mathrm{t}} / n$ and $\eta_{\mathrm{c}, \mathrm{BL}}$ the time-dependent occupation of the conduction level per charge carrier and the occupation of the conduction level per charge carrier in the Boltzmann limit, respectively. For calculating $p_{\mathrm{c}}(t)$, standard drift-diffusion device modeling may be used in conjunction with the MT model. ${ }^{6}$

In general, the calculation of $p_{\mathrm{c}}(t)$ at each position is an integral part of the calculation. However, in admittance experiments the density modulation is small and sinusoidal, so that the ac contribution to $J$ follows already from the frequency-dependent mobility,

$$
\mu_{\mathrm{ac}}\left(\omega, n_{0}\right) \equiv \frac{J_{\mathrm{ac}}\left(\omega, n_{0}\right)}{e n\left(\omega, n_{0}\right) F} .
$$

The functions $J(\omega)$ and $n(\omega)$ are the Fourier transforms of the current density $J(t)=J(\infty)+J_{\mathrm{ac}}(t)$ and the carrier density $n(t)=n_{0}+n_{\mathrm{ac}}(t)$, respectively, for the case of an infinitesimal density increase at $t=0$ in a uniform system.

\section{APPLICATION: $C(V)$ CURVES OF PF-TAA-BASED DEVICES}

In Fig. 4, the approach developed in the previous section is applied to 97- and 121-nm-thick PF-TAA-based devices. The $C(V)$ curves were obtained using a small-signal ac extension $^{39}$ of a drift-diffusion device model, ${ }^{45}$ within which the (generally complex) ac mobility as given by Eq. (7) has been added to the real dc mobility. Within the model, the current density includes a diffusion contribution as described by the generalized Einstein equation (see Appendix A of Ref. 45). We note that the use of small-signal methods for calculating $C(V)$ curves is widespread (see, e.g., Ref. 46) and that the approach for including a frequency-dependent mobility such as that developed in this paper can be used straightforwardly in conjunction with such methods.

For the 97-nm devices, the experimental results are well described using $E_{\mathrm{c}}=-0.75 \sigma$, the value used in Fig. 3. The $C(V)$ curves are quite sensitive to $E_{\mathrm{c}}$, as may be seen from the dashed curves in Fig. 4(a) which give the results at $100 \mathrm{~Hz}$ for $E_{\mathrm{c}}=-0.5 \sigma$ and $-1.0 \sigma$. A fair agreement of the highvoltage capacitance as a function of $V$ and $f$ with the model predictions is obtained, which is remarkable in view of the large time scale covered by the $\mu(t)$ curves and their sensitivity to $\hat{\sigma}$ and $E_{\mathrm{c}}$. The observed decrease of the relaxation-induced
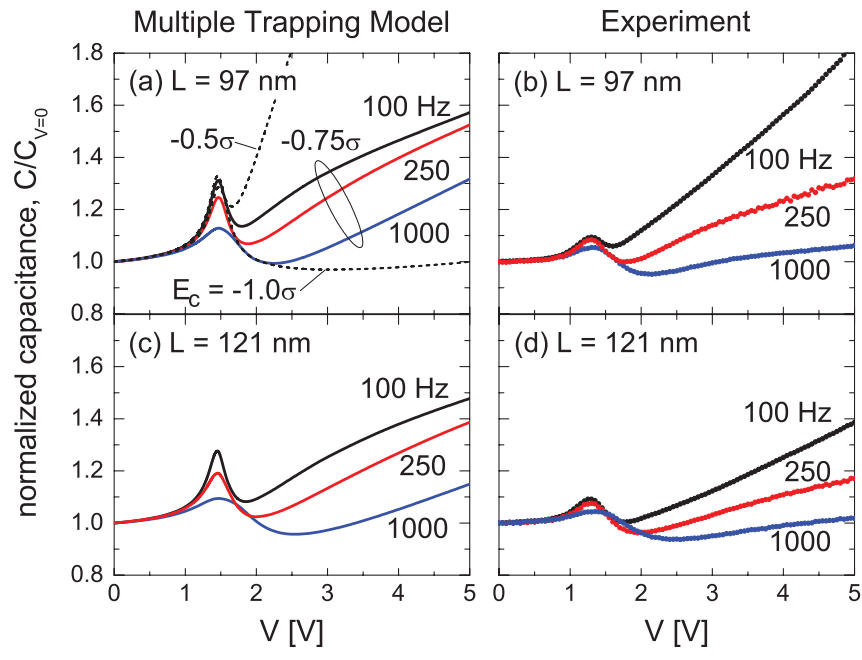

FIG. 4. (Color online) Comparison of theoretical and experimental $C(V)$ curves at $295 \mathrm{~K}$ using $E_{\mathrm{c}}=-0.75 \sigma$, for $(\mathrm{a}, \mathrm{b}) 97-\mathrm{nm}$ and (c,d) 121-nm PF-TAA devices. In (a), the dashed curves show MT results at $100 \mathrm{~Hz}$ for $E_{\mathrm{c}}=-0.5 \sigma$ and $-1.0 \sigma$.

capacitance with increasing layer thickness is found as well from the model. The discrepancy concerning the low-voltage peak height might be due to lateral variations of $V_{\mathrm{bi}}$, leading to peak widening, and indicates that, in future refined models of the electrode boundary conditions should be developed.

It is interesting to note that the modeled relaxation process extends over a time range up to approximately $1 \mathrm{~s}$, for charge carriers in the Boltzmann limit, as may be seen from Fig. 3 or as may be calculated from Eq. (5). This implies that when measuring steady-state $J(V)$ curves great care should be taken concerning the rate of increase of the voltage, even at room temperature. We find that the current-density response to a sudden increase of the voltage can indeed give rise to a timedependent signal which only saturates after $1 \mathrm{~s}$, depending on the detailed initial and final voltage values. The results of these "dark injection" experiments are presented elsewhere.

The relaxation-enhanced capacitance is the net result of various counteracting effects, as may be seen from Fig. 5. Figure 5(a) shows that $\mu_{\mathrm{ac}}$ increases with increasing frequency, but only relatively weakly, so that the capacitance (defined as the imaginary part of $J(\omega) /[\omega V(\omega)]$ ) decreases with increasing frequency. Figure 5(b) gives for a representative example the normal-
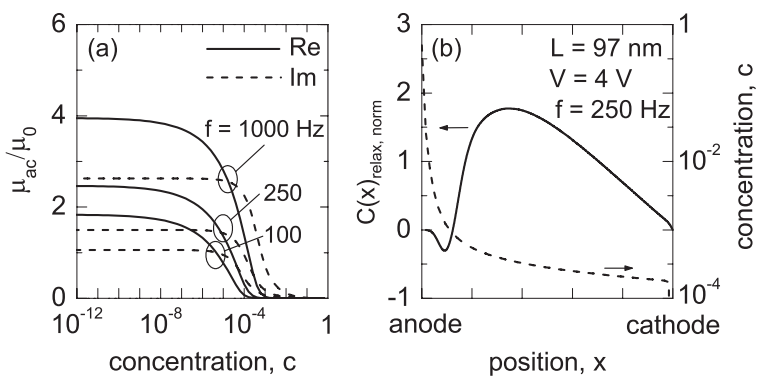

FIG. 5. (a) Calculated concentration dependence of the real and imaginary components of $\mu_{\mathrm{ac}} / \mu_{0}$ for PF-TAA. (b) Local relaxation contribution to the capacitance (normalized, solid curve) and carrier concentration (dashed) for a 97-nm device at $4 \mathrm{~V}$ and $250 \mathrm{~Hz}$. 
ized position-resolved contribution to $C$, obtained by only including relaxation in an infinitesimal position range. We find that its integral is to an excellent approximation equal to the total relaxation contribution. The figure reveals that the relaxation contribution to $C$ is a bulk effect; it is small near the anode, where the carrier concentration is high but the relaxation very fast [see Fig. 5(a)], and near the cathode, where $c$ is small.

\section{SUMMARY AND CONCLUSIONS}

We have shown how the effect of charge-carrier relaxation on the time-dependent mobility in organic semiconductor devices based on materials with a Gaussian DOS may be predictively modeled. For that purpose, three-dimensional Monte Carlo modeling has been used to calculate the effective value of the conduction energy level which enters calculations of $\mu(t)$ within a multiple-trapping model. Assuming hopping in a simple-cubic lattice and assuming Miller-Abrahams hopping rates, we find values of $E_{\mathrm{c}}$ in the range - $(0.5-0.8) \sigma$. A slight dependence on the disorder parameter $\hat{\sigma}$ cannot be excluded. The carrier density dependencies of $\mu(t)$ as obtained from both approaches were found to be in excellent agreement. Quantitative modeling is then possible by making use of the values of the width of the Gaussian DOS and the hopping attempt frequency $\left(\sigma\right.$ and $\left.\nu_{0}\right)$, which can be derived from an analysis of steady-state $J(V)$ curve measurements.
The approach was demonstrated successfully for the specific case of the low-frequency differential capacitance of MOM devices based on the blue-emitting polymer PF-TAA. A fair description of the pronounced relaxation-related contribution to the capacitance was obtained using $E_{\mathrm{c}}=-0.75 \sigma$. It is thus in this case possible to describe the relaxation contribution to the capacitance as an intrinsic effect, which follows directly from considering multiple trapping in the Gaussian DOS, without introducing additional parameters. Future studies should consider the additional contribution to the transient current density due to anomalous dispersive transport, which is expected to be most relevant in relatively thick devices. We envisage applications to the modeling of other transient effects, such as transient electroluminescence, dark current injection experiments, ${ }^{47}$ and hysteretic $J(V)$ measurements.

\section{ACKNOWLEDGMENTS}

W.Chr.G. and J.J.M.v.d.H. contributed equally to this work. The authors thank Sumation Co., Ltd., for the supply of Lumation Blue Series polymers. This research is supported by the Dutch Technology Foundation STW (VIDI Grant No.07575, W.Chr.G.), the Dutch nanotechnology program NanoNed (J.J.M.v.d.H. and S.L.M.v.M.), and the European Community (Program No. FP7-213708 (AEVIOM), P.A.B. and R.C.).

\footnotetext{
*w.c.germs@tue.nl

${ }^{1}$ H. Bässler, Phys. Status Solidi B 175, 15 (1993).

${ }^{2}$ M. C. J. M. Vissenberg and M. Matters, Phys. Rev. B 57, 12964 (1998).

${ }^{3}$ C. Tanase, E. J. Meijer, P. W. M. Blom, and D. M. de Leeuw, Phys. Rev. Lett. 91, 216601 (2003).

${ }^{4}$ W. F. Pasveer, J. Cottaar, C. Tanase, R. Coehoorn, P. A. Bobbert, P. W. M. Blom, D. M. de Leeuw, and M. A. J. Michels, Phys. Rev. Lett. 94, 206601 (2005).

${ }^{5}$ V. Coropceanu, J. Cornil, D. A. da Silva Filho, Y. Olivier, R. Silbey, and J.-L. Brédas, Chem. Rev. 107, 926 (2007).

${ }^{6} \mathrm{H}$. Böttger and V. V. Bryksin, Hopping Conduction in Solids (Akademie-Verlag, Berlin, 1985).

${ }^{7}$ H. Scher and E. W. Montroll, Phys. Rev. B 12, 2455 (1975).

${ }^{8}$ J. C. Scott, L. Th. Pautmeier, and L. B. Schein, Phys. Rev. B 46, 8603 (1992).

${ }^{9}$ P. M. Borsenberger, R. Richert, and H. Bässler, Phys. Rev. B 47, 4289 (1993).

${ }^{10}$ P. M. Borsenberger, E. H. Magin, M. Van der Auweraer, and F. C. De Schrijver, Phys. Status Solidi A 140, 9 (1993).

${ }^{11}$ D. Hertel, H. Bässler, U. Scherf, and H. H. Hörhold, J. Chem. Phys. 110, 9214 (1999).

${ }^{12}$ R. A. Street, K. W. Song, J. E. Northrup, and S. Cowan, Phys. Rev. B 83, 165207 (2011).

${ }^{13}$ N. Christ, S. W. Kettlitz, S. Züfle, S. Valouch, and U. Lemmer, Phys. Rev. B 83, 195211 (2011).

${ }^{14}$ D. Dascǎlu, Solid-State Electron. 11, 491 (1968).

${ }^{15}$ H. C. F. Martens, H. B. Brom, and P. W. M. Blom, Phys. Rev. B 60, R8489 (1999).
}

${ }^{16}$ T. Okachi, T. Nagase, T. Kobayashi, and H. Naito, Jpn. J. Appl. Phys. 47, 8965 (2008); Appl. Phys. Lett. 94, 043301 (2009).

${ }^{17}$ L. M. Andersson and O. Inganäs, Org. Electr. 8, 423 (2007).

${ }^{18}$ R. Richert, L. Pautmeier, and H. Bässler, Phys. Rev. Lett. 63, 547 (1989).

${ }^{19}$ J. Noolandi, Phys. Rev. B 16, 4474 (1977).

${ }^{20} \mathrm{~T}$. Tiedje and A. Rose, Solid State Commun. 37, 49 (1980).

${ }^{21}$ J. Orenstein and M. A. Kastner, Phys. Rev. Lett. 46, 1421 (1981); Solid State Commun. 40, 85 (1981); J. Orenstein, M. A. Kastner, and V. Vaninov, Philos. Mag. B 46, 23 (1982).

${ }^{22}$ J. M. Marshall, Rep. Prog. Phys. 46, 1235 (1983).

${ }^{23}$ G. F. Seynhaeve, R. P. Barclay, G. J. Adriaenssens, and J. M. Marshall, Phys. Rev. B 39, 10196 (1989).

${ }^{24}$ S. D. Baranovskii, H. Cordes, F. Hensel, and G. Leising, Phys. Rev. B 62, 7934 (2000).

${ }^{25}$ V. R. Nikitenko, H. von Seggern, and H. Bässler, J. Phys. Condens. Matter 19, 136210 (2007).

${ }^{26}$ A. V. Nenashev, F. Jansson, S. D. Baranovskii, R. Österbacka, A. V. Dvurechenskii, and F. Gebhard, Phys. Rev. B 81, 115204 (2010).

${ }^{27}$ S. D. Baranovskii, O. Rubel, F. Jansson and R. Österbacka, Adv. Polym. Sci. 223, 45 (2010).

${ }^{28}$ D. Monroe, Phys. Rev. Lett. 54, 146 (1985).

${ }^{29}$ B. Hartenstein, H. Bässler, A. Jakobs, and K. W. Kehr, Phys. Rev. B 54, 8574 (1996).

${ }^{30}$ R. Coehoorn, W. F. Pasveer, P. A. Bobbert, and M. A. J. Michels, Phys. Rev. B 72, 155206 (2005), and references therein.

${ }^{31}$ I. I. Fishchuk, V. I. Arkhipov, A. Kadashchuk, P. Heremans, and H. Bässler, Phys. Rev. B 76, 045210 (2007). 
${ }^{32}$ J. Cottaar, L. J. A. Koster, R. Coehoorn, and P. A. Bobbert, Phys. Rev. Lett. 107, 136601 (2011).

${ }^{33}$ In all experiments, $C_{V=0}$ is very close to the geometrical capacitance, as obtained well below $V=0 \mathrm{~V}$. The relative dielectric permittivity is 3.2 at high frequencies and increases slightly with decreasing frequency ( $\sim 7 \%$ from 2500 to $100 \mathrm{~Hz}$ ) due to dielectric relaxation. ${ }^{15}$

${ }^{34}$ S. L. M. van Mensfoort and R. Coehoorn, Phys. Rev. Lett. 100, 086802 (2008).

${ }^{35}$ S. L. M. van Mensfoort, S. I. E. Vulto, R. A. J. Janssen, and R. Coehoorn, Phys. Rev. B 78, 085208 (2008).

${ }^{36}$ R. J. de Vries, S. L. M. van Mensfoort, V. Shabro, R. A. J. Janssen, and R. Coehoorn, Appl. Phys. Lett. 94, 163307 (2009).

${ }^{37}$ The barrier at the Pd electrode was obtained from the data given in Ref. 35 using the discrete drift-diffusion model employed in the present paper.
${ }^{38}$ R. J. de Vries, S. L. M. van Mensfoort, R. A. J. Janssen, and R. Coehoorn, Phys. Rev. B 81, 125203 (2010).

${ }^{39}$ W. Chr. Germs, S. L. M. van Mensfoort, R. J. de Vries, and R. Coehoorn (unpublished).

${ }^{40}$ D. C. Tripathi, A. K. Tripathi, and Y. N. Mohapatra, Appl. Phys. Lett. 98, 033304 (2011).

${ }^{41}$ N. D. Nguyen, M. Schmeits, and H. P. Loebl, Phys. Rev. B 75, 075307 (2007).

${ }^{42}$ K. K. Tsung and S. K. So, J. Appl. Phys. 106, 083710 (2009).

${ }^{43}$ A. Miller and E. Abrahams, Phys. Rev. 120, 745 (1960).

${ }^{44}$ From Eq. (3) in Ref. 4, with $c_{1}$ equal to the exponential rate reduction factor for nearest-neighbor hopping.

${ }^{45}$ R. Coehoorn and S. L. M. van Mensfoort, Phys. Rev. B 80, 085302 (2009).

${ }^{46}$ E. Knapp and B. Ruhstaller, Appl. Phys. Lett. 99, 093304 (2011).

${ }^{47}$ D. Poplavskyy, W. Su, and F. So, J. Appl. Phys. 98, 014501 (2005). 